\title{
Avian Infectious Bronchitis Virus in Brazil: A Highly Complex Virus Meets a Highly Susceptible Host Population
}

\section{author(s)}

Brandão PE

Coronavirus Research Group Department of Preventive Veterinary Medicine and Animal Health

School of Veterinary Medicine

University of São Paulo, Brazil.

\section{Mail Address}

Prof. Dr. Paulo Eduardo Brandão.

Departamento de Medicina Veterinária Preventiva e Saúde Animal

Faculdade de Medicina Veterinária e Zootecnia

Universidade de São Paulo

Av. Prof. Dr. Orlando M. Paiva, 87

05.508-270. São Paulo, SP, Brazil.

E-mail: paulo7926@usp.br

\section{Keywords}

$\mathrm{IBV}$, genealogy, pathogeny, epidemiology.

\section{ABSTRACT}

Infectious bronchitis (IB) is a highly aggressive disease for poultry in terms of symptoms and economic losses, and the control of this disease is difficult if flocks are not protected against type-specific challenges by the Avian infectious bronchitis virus (IBV). This article summarizes data presented by the author at the Workshop on Infectious Bronchitis 2009 on IB and IBV, including future developments on the field.

\section{INTRODUCTION}

Back in 1931, Schalk \& Hawn (1931) investigated an outbreak of respiratory disease in birds in the USA, and after a thorough and interesting investigation, they ruled out bacterial or toxic origins of the disease, as the causative agent was filterable, differently from bacterial diseases, and transmissible between birds, which is not expected in intoxication. Though the virus itself was not described then, they produced an inspiring article, in which the authors name the disease infectious bronchitis (IB).

The causative agent, Avian infectious bronchitis virus (IBV) became then the first identified coronavirus, which is now classified as a member of Group 3 of the genus Coronavirus (Gammacoronavirus) (Nidovirales: Coronaviridae), with dozens of recognized serotypes and a vast range of pathotypes.

The identification of serotype and pathotypes determine host-parasite relationship levels between IBV and its natural host Gallus gallus, whereas there may be different and hard-to-predict clinical manifestations depending on the pathotype that infects birds with a classically described negative relationship between protection and distance for the spike (S) protein.

$\mathrm{IBV}$ vaccination is currently the biggest concern of the Brazilian poultry industry, as the Massachusetts is the only serotype allowed to be used in live attenuated vaccines by the Brazilian Ministry of Agriculture. The reason for concern is that, as vaccination with a live strain provides a much more efficient immunity in primo vaccinations, the Massachusetts monotype vaccine is not able to provide protection fastly and broadly enough to make chickens able to circumvent IBV challenges in Brazil, where genotypes are highly divergent from this vaccine type, leaving one of the world's largest chicken populations vulnerable.

The aim of this article is to review some data on IBV and IB in Brazil gathered specifically by the Coronavirus Research Group of the University of São Paulo, Brazil. This review was presented by the author at the Workshop on Infectious Bronchitis held by APINCO in 2009, based on some results published in meetings and conferences by the Group, as well as some insights on that matter and on the basis for the control of the disease in Brazil. 


\section{QUESTIONS IN STUDIES ON DISEASES IN POPULATIONS}

One of the most common questions in studies on diseases in populations (i.e. epidemiology) is the simple frequency of occurrence, and its incidence from a geographical point of view. This information is highly significant to show the importance of a disease and to make allocation of resources to its control.

Sandri et al. (2009) showed that $73.5 \%$ of 200 flocks (breeders, layers, and broilers) were positive for IBV, using a classic RT-PCR targeted to the $3^{\prime}$ untranslated region (UTR) of the virus genome, a highly-preserved region amongst IBVs. Frequencies ranged from $85.42 \%$ in broilers to $63.64 \%$ in layers, with $74.24 \%$ in breeders.

In that same report, it was shown that not only IBV is present in high frequencies in the Northeastern, Central-Western, Southeastern, and Southern regions of Brazil, but also that non-Massachusetts genotypes prevail, independently of bird type (breeder, layer or broiler), at a frequency as high as $77.55 \%$.

These results show that non-Massachusetts types, which may be called variants, and that do not belong to any of the known IBV types, may be responsible for more than three quarters of IB occurrence in Brazil.

A further question is: How do Brazilian IBVs distribute in the different organs of chickens presenting different clinical signs related to IB, such as respiratory, renal, enteric and reproductive (in both males and females) symptoms?

Sandri et al. (2009) have addressed this question and - interestingly, but not surprisingly - IBV RNA was found in organs such as lungs, tracheas, kidneys, female reproductive organs, testes, spleen, liver, heart, and bursa and, predominantly, in enteric contents. The pathogenic significance of some of such findings is still controversial, but the authors have dedicated themselves to shed more light on this question.

From a quantitative point of view, these results on the epidemiology (or ornithoepidemiology, in this case) of $I B$ are totally new and extremely valuable due to the large number of samples collected, but the prevalence of non-Massachusetts types and the diversity of diseases associated to IBV in Brazilian poultry is not new. Quoting Erich Maria Remarque, up to this point it's "All quiet on the Western front".

Currently, sequence data banks, such as the Genbank, include plenty of IBV sequences, mostly from the spike gene. In order to improve the interpretation of nucleotide and amino acid sequences, the larger the number of variables associated to a given set of sequences, the broader the patterns that can be depicted.

From an epidemiological point of view, this can be illustrated by molecular epidemiology, where sequence data are associated to classic data from epidemiological measurements and observations, aiming, for instance, at backtracking of outbreaks and determining sources of IB infections.

One of the studies carried out by the Coronavirus Research Group, but not yet published, refers to the detection of avian coronavirus in quails in a farm where layer chickens and quails were kept in closely located facilities. Layer chickens presented IB-like symptoms at the time of sampling, and quails had eggshell malformations and severe oviduct edema. This indicates that quails may be reservoirs of IB infecting chickens, and this may be an even bigger problem when one takes into account that quails are not vaccinated against IB. Further studies are under way to isolate this virus and sequence some of its genes.

\section{FINAL REMARKS}

Infectious bronchitis virus, as all coronaviruses, is highly complex in terms of replication, pathogeny and genetics, but the diversity of types makes it the most intricate member of the genus. Considering this complexity, a further question is: how do the Brazilian IBV strains relate to each other and to other IBVs from a genealogic standpoint, and how many strains of this virus exist in Brazil?

After sequencing partial spike genes of 20 IBV strains collected from breeders, broilers, and layers with IBlike symptoms, Brandão et al. (2009) described three sub-strains in an exclusively Brazilian IBV genotype, genetically distant from the Massachusetts type. Unexpectedly, two strains were typed as 4/91 (also known as 793B and (R88), showing that IBV diversity in Brazil is higher than previously believed.

In addition, some Massachusetts strains detected in Brazil are different from the original Massachusetts strain used for vaccination (Villarreal et al., 2008). Although it is not possible to definitely conclude that these were wild virus strains, these results show that Massachusetts IBVs are present and evolving in Brazilian flocks.

All these processes must be considered as hostparasites relationships. When two biologic systems meet, such as a virus and its host cell, the interaction between their molecules both guides and is guided by 
evolution, and the next generation will present detectable traces of this interaction. One of these traces is the type of selection, which may be purifying (when the rate of synonymous nucleotide substitutions, i.e., those that do not lead to amino acid changes, is higher than the non-synonymous substitutions), positive (when the rate is higher for non-synonymous substitutions), or even neutral.

In the same aforementioned study, Brandão et al. (2009) detected a slightly positive selection in some of the Brazilian IBVs, indicating that the hosts are not able to suppress amino acid evolution, and that low immunity is a strong candidate as a responsible for this pattern.

A further complexity of coronaviruses is the "slippery" nature of their replicase, which may change from the template RNA from a strain into that from another strain during genomic RNA replication if COinfection occurs. The result is then a recombinant strain.

Recombination was detected amongst Brazilian genotype strains (Brandão et al., 2009), but, strikingly, the donor of a small recombinant fragment is a still non-identified IBV strain. On the other hand, no recombination was detected to date between Brazilian genotype strains and a vaccine strain. Isolation of that recombinant IBV is being attempted by the authors in order to obtain further data on its genome.

IBV is undoubtedly one of the most important pathogens of poultry, and the use of gene molecular biology for the development of a diagnostic tool has allowed important advances in the fields of IB epidemiology and control. RT-PCR assays for IBV detection are based on the detection of viral RNA in samples, allowing fast and accurate diagnosis from the populational point of view, which is the main interest of poultry industry.

Nonetheless, RT-PCR assays used to date, despite their considerable value for diagnosis, do not allow differentiating viral replication in a given tissue from the mere presence of genomic, non-replicative RNA in that tissue in an individual bird

When replicating, viruses make the host cell synthesize viral protein by means of messenger RNAs (mRNAs) transcription. In Coronavirus, mRNA transcription characteristically produces sub genomicsized mRNAs for each protein to be synthesized, all marked at the $5^{\prime}$ end with a same sequence, called leader RNA, regardless the mRNA in question. Using this knowledge, an RT-PCR assay was described (Brandão et al.; 2009), targeting both the leader and the $5 \mathrm{a}$ non-structural proteins of IBV, and the amplification was only possible when the leader and the 5 a sequences were close enough, and only happened when the mRNA for this protein was present. In other words, a positive result means that mRNA is being transcribed, and therefore, that IBV is replicating in that tissue. Coronavirus mRNA transcription is indeed complex, and it is one of the factors of the success of this genus in a wide range of hosts.

The most desired answers from the poultry industry's standpoint is how to suppress IBV replication by means of antibodies that are specific enough to prevent viral adsorption or spread. In the future, one of the answers may be to defeat the virus inside the cell. This could be achieved by the use of RNA interference (RNAi), which finding was awarded the 2006 Nobel Prize in Medicine. Briefly, if a synthetic double-stranded RNA, which antisense strand is complementary to a given mRNA present in the cytoplasm, is introduced into or generated inside the cytoplasm, a chain of reactions mediated by the cellular RNA-induced silencing complex (RISC) will ultimately lead to the degradation of that specific mRNA and no protein will be synthesized. In other words, it is possible to turn off protein synthesis by "killing the messenger".

The Coronavirus Research Group has a project on the development of RNAi technologies for the inhibition of IBV replication. The target is precisely the leader RNA sequence, which is common in every IBV mRNA, meaning that not only one, but all of its mRNAs will broken down in the cytoplasm, which has already been described to SARS coronavirus. Therefore, the power of coronaviruses becomes now its Achilles' heel.

There is still a lot of work to do. Brazilian research groups on IBV and IB, both in basic and applied fields, are at the same level of other groups worldwide, with promising results and projects. People involved in serious research in this area in many Brazilian research centers have a solid theoretical background and the technical skills to explore the large ocean of questions to be answered, as Schalk and Hawn did more than seven decades ago.

\section{REFERENCES}

Brandão PE, Sandri TL, Souza SP, Kuana SL, Richtzenhain LJ, Villarreal LYB. Recombination, point mutations and positive selection on the basis of the molecular diversity of Brazilian strains of Avian infectious bronchitis virus. Proceedings of the $6^{\text {th }}$ International Symposium on Avian Corona- and Pneumoviruses and Complicating Pathogens; 2009; Rauischholzhausen. Germany. p.47-58.

Brandao PE, Villarreal LYB, Souza SP, Sandri TL, Ayres G, Richtzenhain 
LJ. A reverse-transcription polymerase chain reaction for the detection of avian infectious bronchitis virus (IBV) replication based on mRNA for the $5 a$ protein of the virus. Proceedings of the 20th National Meeting of Virology; 2009; Águas de São Pedro. São Paulo. Brasil. p.28

Sandri TL, Villarreal LYB, Souza SP, Peruzzetto MC, Richtzenhain LJ, Brandão PE. Geografic and tropism patterns on the occurrence of variant genotypes of avian infections bronchitis virus in Brazilian poultry. Anais da Conferência APINCO 2008 de Ciência e Tecnologia Avícolas; 2008; Santos. São Paulo. Brazil. p. 229.

Schalk AF, Hawn MC. An apparently new respiratory disease of baby chicks. Journal of the American Veterinary Medicine Association 1931; 78:413-416.

Villarreal LYB, Sandri TL, Balestrin G, Rocha P, Richtzenhain LJ, Brandão PE. A comparison between Field and vaccine strains of Massachusetts serotype of avian infectious bronchitis virus. Anais da Conferência APINCO 2008 de Ciência e Tecnologia Avícolas 2008; Santos, São Paulo. Brasil. p. 237. 